\title{
Monophagous olive moth and its bacterial microbiota: unveiling the holobiont towards pest management
}

\section{Tânia Nobre}

To cite this article: Tânia Nobre (2020): Monophagous olive moth and its bacterial microbiota: unveiling the holobiont towards pest management, Biocontrol Science and Technology, DOI: 10.1080/09583157.2020.1842327

To link to this article: https://doi.org/10.1080/09583157.2020.1842327

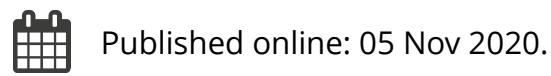

Submit your article to this journal ¿

Q View related articles $\square$

View Crossmark data ¿ 


\title{
Monophagous olive moth and its bacterial microbiota: unveiling the holobiont towards pest management
}

\author{
Tânia Nobre \\ MED - Mediterranean Institute for Agriculture, Environment and Development, University of Évora, Évora, \\ Portugal
}

\begin{abstract}
Symbiotic-based pest management strategies are promising but require previous knowledge on the microbial community structure and on key microorganisms. Highly specialised pests, as the monophagous olive moth, are likely to have co-evolved microbiota that aid in overcoming specific plant defences that have emerged from the tight insect-plant interaction. Here we report a first attempt to identify olive moth bacterial microbiome based on $16 \mathrm{~S}$ sequencing. This preliminary analysis intended to highlight potential host-symbiont interactions, representing key aspects in the moth survival and adaptation, such as nutrition (e.g. Pseudomonas sp.), detoxification (e.g. Acetinobacter sp. or Staphylococcus sp.), and protection/defence (e.g. Actinobacteria).
\end{abstract}

\section{ARTICLE HISTORY}

Received 22 June 2020

Accepted 20 October 2020

\section{KEYWORDS}

Prays oleae; microbiota; symbiotic-based strategies; pest management

Natural enemies of pest are traditionally seen as parasites, pathogens, and predators that have the capacity of managing pests and their damage. However, this view can be enlarged to encompass the pest associated microbiota, as these microorganisms cannot be classified exclusively as beneficial, harmless or deleterious because their impact on the insect pest can depend on circumstance. Exploring this (in)balance is one way that a symbiotic-based pest management strategy can develop. A pre- requisite for such an approach is the knowledge of the microbiota associated with the insect pest and its determinants.

The olive moth, Prays oleae (Bernard, 1978) (Lepidoptera, Praydidae) remains an abundant insect pest of olive trees of which not many natural enemies are known or exploited. It is a monophagous species with three annual generations, each one developing at a different organ from its olive host (Olea europaea L.): leaves (phillophagous generation), flowers (antophagous generation) and fruits (carpophagous generation). Depending on the olive as only host for survival and reproduction, the olive moth had to evolve to counterpart the wide variety of secondary metabolites that the plant produces as a strategy of chemical defence to tolerate biotic (and abiotic) stresses. Amongst them, the olive contains several unusual secoiridoids commonly known as oleosides that are unique to Oleaceae, which the most significant is the oleuropein. This compound has been detected in various organs of the olive tree, like leaves, flowers, bark, and roots, 
but it is more known from the fruit, being responsible for its bitter taste. Other phenolics compounds, like rutin, tannin, cafeic, verbascoside and luteolin are suggested to be involved in the defence against Prays oleae (Ilias et al., 2017). To be able to use olive nutrients, herbivores and microbes need to deal with abundant secondary metabolites. For example, the olive fruit fly -Bactrocera oleae (Rossi, 1790) (Diptera, Tephritidae)evolved to harbour a vertically transmitted and obligate bacterial symbiont, the Candidatus Erwinia dacicola, that allows the insect to cope with the abundant secondary metabolites and in particularly, with the oleuropein. It is unknown how the olive moth overcome the plant host defences. It is now widely accepted that holobionts -host organism and its symbiotic microbiota- are widespread in nature, making these entities the likely unit of selection and evolution. Hence the importance of addressing the olive moth and its microbiota as a whole: the selection pressure to overcome plant defensive compounds is exerted not only on the insect but also on the associated core bacteria. Here we report a first attempt to identify Prays oleae microbiome based on 16S sequencing, in search for potential symbionts that would aid in detoxification considering the plants defensive compounds. This knowledge is the first step towards identification and selection of symbionts that can be further used as a potential tool for population control.

Olive fruits were collected in September 2019, in Crete, Greece in a single olive orchard and the olives were brought into the laboratory and left in vials at room temperature until adult emergence (corresponding to the carpophagous generation). Individuals were stored at $-20^{\circ} \mathrm{C}$ in $70 \%$ ethanol until DNA extraction. Extraction was done per individual using the DNA Zymobiomics kit (Zymo Research $\left.{ }^{\circledast}\right)$ and following the advised protocol. The NGS sequencing procedures, for the three insects independently, were performed at STABVIDA, Lda. (Portugal). After quality control of the DNA, to ensure samples had sufficient integrity and quantity for optimal amplification, the library construction was performed using the Illumina 16S Metagenomic Sequencing Library preparation protocol and the generated DNA fragments (DNA libraries) were sequenced with MiSeq Reagent Kit v3 in the lllumina MiSeq platform, for the $\mathrm{V} 3$ and V4 regions of the 16S rRNA gene, using $300 \mathrm{bp}$ paired-end sequencing reads (available at NCBI, BioProject ID PRJNA640599). The analysis of the generated raw sequence data was carried out using QIIME2 v2019.10. Data denoising was done using the DADA2 plugin by trimming and truncating low quality regions, dereplicating the reads and filtering chimeras. The scikit-learn classifier was used to train the classifier using the SILVA (release 132 QIIME) database, with a clustering threshold of $97 \%$ similarity. For classification purposes, only OTUs containing at least 10 sequence reads were considered as significant.

The samples generated from 117,020-259,912 raw sequence reads, which is in accordance with the expected output. After denoising, a total of 1,017 unique features (OTUs) of 221 bp could be detected (with a total frequency of 170,771 in the three samples, being a sample defined by a single adult of olive moth). Even though the total amount of OTUs observed per sample varied considerably (also initial DNA concentration and integrity was different between samples, which might have led to this output differences), the alpha-rarefaction curve clearly reached a plateau indicating that the sequencing was deep enough to detect present $16 \mathrm{~S}$ diversity (Figure 1). Almost all reads (99.35\%) were associated with Bacteria. 


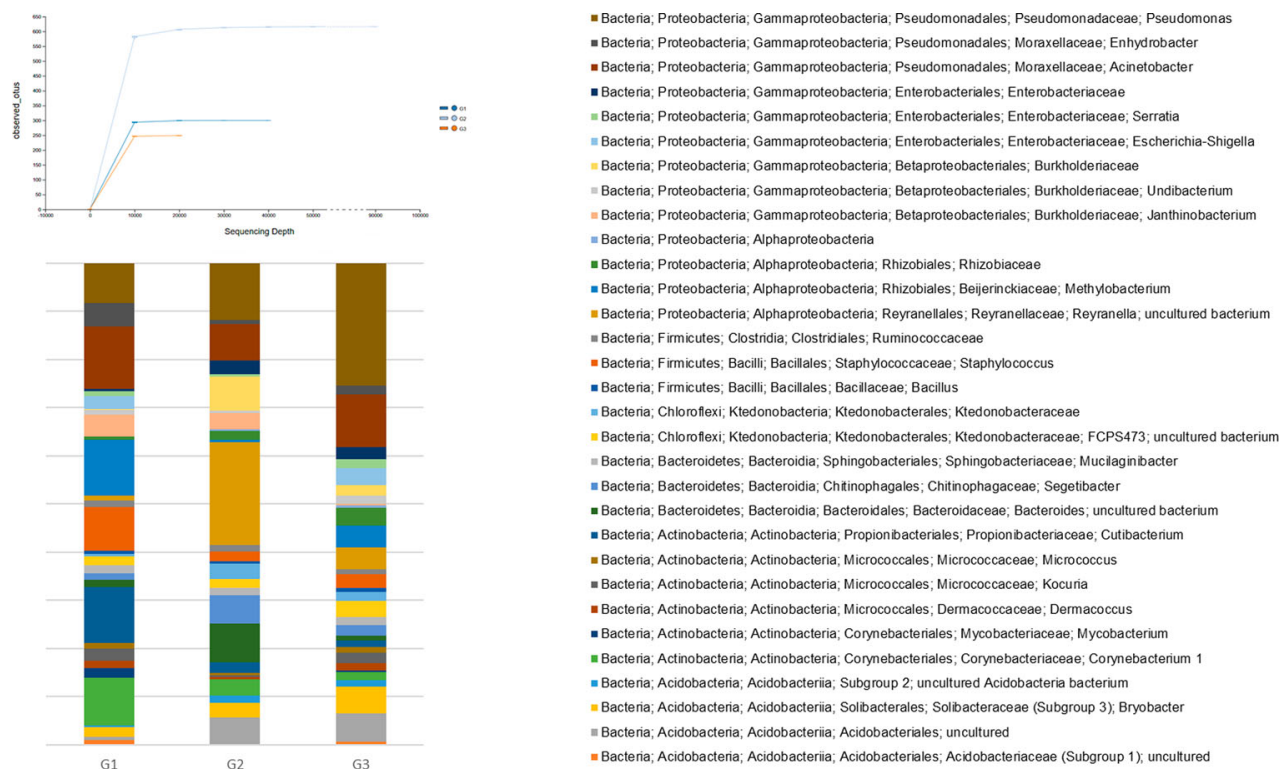

Figure 1. Observed OTUs Alpha rarefaction curve and taxonomic identification of the shared OTUs per Prays oleae sample.

Defining, conservatively, the core microbiota as the bacteria present in all 3 samples with more than 10 reads per sample, 31 classified groups emerged (Figure 1). A high number of reads correspond to non-classified bacteria -the most frequent OTU $(11,056)$ was classified to the Bacteria level only using the SILVA (with a $98 \%$ similarity), as well as 16 others shared OTUs. The most frequent bacteria was classified as Pseudomonas sp., a ubiquitous genus with high metabolic diversity, often encountered associated to insects as an entomopathogen, but also showing a high metabolic versatility with amylolytic, cellulolytic, xylanolytic, lipolytic, and esterase activities (Jing et al., 2020). Also, there are references to Pseudomonas sp. aiding in detoxification processes including degradation of insecticides. Still within the Pseudomonadales, but belonging to Moraxellaceae, the presence of Acinetobacter sp. (5949 reads) and Enhydrobacter sp. (1442 reads) should be referred. Acinetobacter are commonly found in several systems and are reported by their capacity to degrade large molecules, including pesticides (Geiger et al., 2009). Enhydrobacter, like Pseudomonas and Acinetobacter, has also been assigned to the core microbiota of other insect pest moths.

Pseudomonas, together with Bacillus, Staphylococcus, Enterobacter, and Enterococcus, are the most widespread bacterial genus in lepidopteran species (Voirol et al., 2018). Surprising enough, no OTU was classified as Bacillus, and the main Firmicutes found in the three samples was a Staphylococcus (2767 reads in total, considering 9 OTUs with only 10 polymorphic sites). Staphylococcus sp. was found to provide resistance to specific toxic compounds - alkaloids and latex- in two lepidopterans (Vilanova et al., 2016). Nonetheless, they are widespread in insects and have been traditionally considered generalist bacteria, highly prevalent in the Lepidotera families Sphingidae and Noctuidae (Visôtto et al., 2009). As to the Enterobacteriaceae, two OTUs could be found in all samples: one classified as Escherichia-Shigella (1008 reads) and a Serratia sp. (543 reads). Other 
Enterobacteriaceae were found comprising 9 OTUs (2754 reads in total). Species of the genus Serratia are known to be associated with insects as pathogens (Inglis \& Lawrence, 2001) and as symbionts (Latorre et al., 2012), or its presence in insect's digestive tract can just be of plant/environmental origin.

Two Alphaproteobacteria are predominantly present in the three samples: Reyranella sp. (6493 reads) and Methylobacterium sp. (3054 reads). The first is associated with different soils, including agricultural and can thus associate to the insect's digestive tract (albeit establishing during oviposition and in olive development, as the adults were captured upon ecloding in laboratory conditions). The second, a methane-utilizing bacterium, has ubiquitous occurrence on plant leaves and has been detected on table olives (Cocolin et al., 2013).

The Actinobacteria are knonw for protective insect-actinobacteria associations, and symbiotic relations might be more common than previously thought. In Prays oleae, five actinobacteria species were present in all samples: in the Propionibacteriales, Cutibacterium sp. (3142 reads); in the Corynebacteriales, Corynebacterium sp. (3126 reads) and Mycobacterium sp. (479 reads); and in the Micrococcales, Kocuria sp. (884 reads), Dermacoccus sp. (599 reads), Micrococcus sp. (475 reads). Also deserving reference is the presence of a Bacteroides in all samples (2570 reads).

This preliminary analysis of the olive moth bacterial microbiota intended to highlight potential host-symbiont interactions, representing key aspects in the moth survival and adaptation, such as nutrition (e.g. Pseudomonas sp.), detoxification (e.g. Acetinobacter sp. or Staphylococcus sp.), and protection/defence (e.g. Actinobacteria). A comprehensive study is now needed, including looking for a conserved microbiota across the different developmental stages of Prays oleae of the three generations developing on the olive tree followed by a functional profiling of the core microbiome. This is a necessary condition for the delineation of a potential pest management strategy based on symbiosis. Arora and Douglas (2017) provided a critical review of multiple pest management approaches available targeting the insect as an holobiont, founded on manipulation of microbial partners. These approaches have been gaining recognition over time, becoming more relevant in the context of modern sustainable agriculture.

\section{Disclosure statement}

No potential conflict of interest was reported by the author(s).

\section{Funding}

This research was supported by the FCT - Fundação para a Ciência e a Tecnologia, I.P., under the project PTDC/ASP-PLA/30650/2017.

\section{Data availability statement}

The data that support the findings of this study are openly available in NCBI with the code BioProject ID PRJNA640599. 


\section{ORCID}

Tânia Nobre (D) http://orcid.org/0000-0002-1855-7451

\section{References}

Arora, A. K., \& Douglas, A. E. (2017). Hype or opportunity? Using microbial symbionts in novel strategies for insect pest control. Journal of Insect Physiology, 103(August), 10-17. https://doi. org/10.1016/j.jinsphys.2017.09.011

Cocolin, L., Alessandria, V., Botta, C., Gorra, R., Filippis, F. D., Ercolini, D., Rantsiou, K., Agraria, D., \& Ii, F. (2013). NaOH-Debittering induces changes in bacterial ecology during table olives fermentation. PLoS One, 8(7), e69074. https://doi.org/10.1371/journal.pone.0069074

Geiger, A., Fardeau, M.-L., Grebaut, P., Vatunga, G., Josénando, T., Herder, S., Cuny, G., Truc, P., \& Ollivier, B. (2009). First isolation of Enterobacter, Enterococcus, and Acinetobacter spp. As inhabitants of the tsetse fly (Glossina palpalis palpalis) midgut. Infection, Genetics and Evolution, 9, 1364-1370. https://doi.org/10.1016/j.meegid.2009.09.013

Ilias, F., Medjdoub, K., \& Gaouar, N. (2017). Phenolics compounds of olive and olive leaves identified in the resistance of Prays oleae (Bernard). Plant Archives, 17(2), 1761-1764.

Inglis, A. G. D., \& Lawrence, A. M. (2001). Effects of Serratia marcescens on the F 1 generation of laboratory-reared Heliothis virescens (Lepidoptera: Noctuidae). Journal of Economic Entomology, 94(2), 362-366. https://doi.org/10.1603/0022-0493-94.2.362

Jing, T., Qi, F., \& Wang, Z. (2020). Most dominant roles of insect gut bacteria: Digestion, detoxification, or essential nutrient provision? Microbiome, 8, 38. https://doi.org/10.1186/s40168020-00823-y

Latorre, A., Lamelas, A., \& Manzano-Mar1, A. (2012). Comparative genomics of Serratia spp.: Two paths towards endosymbiotic life. PLoS One, 7(10), e47274. https://doi.org/10.1371/journal. pone.0047274

Vilanova, C., Baixeras, J., Latorre, A., \& Porcar, M. (2016). The generalist inside the specialist: Gut bacterial communities of two insect species feeding on toxic plants are dominated by Enterococcus sp. Frontiers in Microbiology, 7(June), 1-8. https://doi.org/10.3389/fmicb.2016. 01005

Visôtto, L. E., Oliveira, M. G. A., Ribon, A. O. B., Mares-Guia, T. R., \& Guedes, R. N. C. (2009). Characterization and identification of proteolytic bacteria from the gut of the Velvetbean Caterpillar (Lepidoptera: Noctuidae). Environmental Entomology, 38(4), 1078-1085. https:// doi.org/10.1603/022.038.0415

Voirol, L. R. P., Frago, E., Kaltenpoth, M., Hilker, M., \& Fatouros, N. E. (2018). Bacterial symbionts in lepidoptera: Their diversity, transmission, and impact on the host. Frontiers in Microbiology, 9(Mar), 1-14. https://doi.org/10.3389/fmicb.2018.00556 